\title{
Evaluation of Colorimetric BCA-Based Quantification of Hydrazide Groups on Magnetic Particles
}

\author{
Zuzana Svobodova $\mathbb{D}^{1},{ }^{1}$ Barbora Jankovicova $\mathbb{D}^{1},{ }^{1}$ Zdenek Plichta, $^{2}$ Daniel Horak, ${ }^{2}$ \\ and Zuzana Bilkova iD ${ }^{1}$ \\ ${ }^{1}$ Department of Biological and Biochemical Sciences, Faculty of Chemical Technology, University of Pardubice, Studentska 573, \\ 53210 Pardubice, Czech Republic \\ ${ }^{2}$ Institute of Macromolecular Chemistry, Academy of Sciences of the Czech Republic, Heyrovsky Sq. 2, 16206 Prague 6, Czech Republic
}

Correspondence should be addressed to Zuzana Bilkova; zuzana.bilkova@upce.cz

Received 27 December 2017; Revised 12 March 2018; Accepted 14 March 2018; Published 17 April 2018

Academic Editor: Nikša Krstulović

Copyright ( 2018 Zuzana Svobodova et al. This is an open access article distributed under the Creative Commons Attribution License, which permits unrestricted use, distribution, and reproduction in any medium, provided the original work is properly cited.

\begin{abstract}
Magnetic micro- and nanoparticles (MPs) are considered to provide excellent solid support for many immunoanalytical and bioaffinity applications, particularly when they contain hydrazide groups available for site-specific immobilization of various glycoproteins, such as immunoglobulin $G$ and enzymes. To prepare a highly active bioaffinity carrier with sufficient binding capacity, knowledge as to the type and concentration of functional groups used for ligand binding is crucial. Described here is a simple, nontoxic method for rapid estimation of hydrazide functional groups bound to MPs using bicinchoninic acid (BCA). BCA kits are routinely used for colorimetric detection and quantification of total protein in liquid samples. In this study, the BCA reagent was applied for quantification of hydrazide groups on MPs. The approach was carried out using an adipic acid dihydrazide $(\mathrm{ADH})$ solution and subsequently using various hydrazide-containing magnetic and nonmagnetic carriers differing in the density of hydrazide groups. The BCA test's results obtained on the MPs were compared with those from conventional amino and hydrazide group quantification by the 2,4,6-trinitrobenzenesulfonic acid (TNBS) test.
\end{abstract}

\section{Introduction}

Interest in covalent binding of proteins to planar or spherical solid supports is associated with the rapid development of new affinity carriers and enzyme catalysts [1]. In particular, magnetic micro- and nanoparticles (MPs) are increasingly considered solid supports suitable for many immunoanalytical and bioaffinity applications, such as isolation and purification of target biomolecules [2-4], diagnostics [5, 6], and efficient therapy involving drug delivery [7]. One of the basic premises for such applications is a stable, preferably covalent, linkage between the solid support and the ligand to be immobilized. It has been repeatedly proven that controlled orientation of the coupled ligand is one of the factors improving the molecular recognition and binding efficiency of molecules to be captured. One widely used coupling method involves site-specific immobilization of glycoproteins (enzymes, hormones, and/or antibodies) through their carbohydrate moieties on hydrazide solid supports $[8,9]$. In the case of antibodies, such as immunoglobulin $\mathrm{G}$ (IgG), carbohydrate residues are localized on the Fc fragment which is not involved in antigen recognition. As a result, the fragment antigen-binding (so-called "Fab") remain sterically available for specific reaction with the antigen as the target molecule [10]. The efficiency of such immunomagnetic carriers is considered to be nearly identical to that of biotinstreptavidin systems, yet they are simple and cost-effective. Moreover, they are 2.5 times more efficient than is random coupling of IgG molecules via amino groups [11].

Proper estimation of the particle/protein ratio and concentration of the protein to be immobilized in the binding buffer with the proper amount of MPs are crucial for effective and low-cost coupling. The surface of the MPs must contain a sufficient density of uniformly distributed functional 


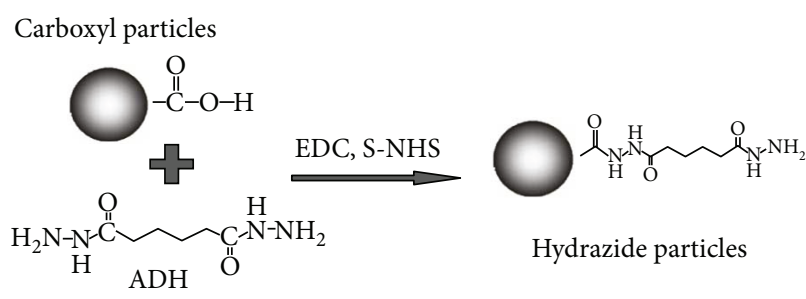

(a)

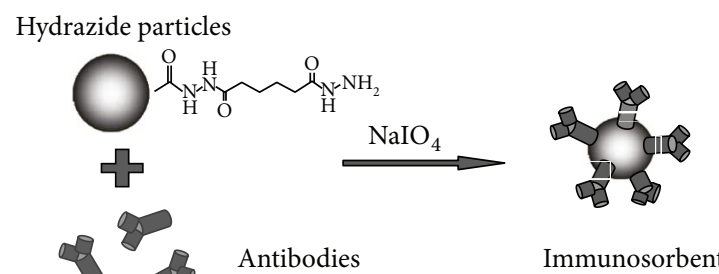

(b)

FIGURE 1: Scheme for covalent coupling of (a) carboxyl-containing MPs with ADH and (b) subsequent site-specific immobilization of IgG via hydrazide groups on MPs.

groups. Information about this density is often not available, and so subsequent evaluation is necessary. The conventional method for amino and hydrazide group quantification includes the 2,4,6-trinitrobenzenesulfonic acid (TNBS) test, which comprises three steps involving work with toxic reagents such as TNBS and methanol. Moreover, the procedure requires approximately $3 \mathrm{~h}$ to complete.

Therefore, an easy, rapid, low-cost, and low-toxic method for quantifying hydrazide groups on magnetic carriers based on bicinchoninic acid (BCA) is needed. This one-step procedure requires only $1 \mathrm{~h}$ incubation at $60^{\circ} \mathrm{C}$ with $\mathrm{BCA}$ containing $\mathrm{Cu}^{2+}$ ions. BCA kits are routinely used for the colorimetric detection and quantification of total protein. In this procedure, $\mathrm{Cu}^{2+}$ ions are reduced by protein to $\mathrm{Cu}^{+}$, which reacts with two BCA molecules to produce a purple end-product absorbing light at $562 \mathrm{~nm}[12,13]$. In this report, a BCA kit is employed in an alternative way for the determination of substances that normally interfere with BCA during total protein determination [14]. Such application was first described by Kakabakos et al. $[15,16]$ who used BCA for the determination of various functional groups, such as sulfhydryl, aldehyde, hydrazide, $N$-hydroxysuccinimide, and/or tyrosine, thyroxine, or cysteine on Sepharose. The method has been applied also for the determination of amino groups [17].

The aim of this work was to confirm that this method is suitable for the determination of hydrazide functional groups on the surface of MPs. Control experiments were performed with adipic acid dihydrazide $(\mathrm{ADH})$ in solution, where the amount of hydrazide groups was known. Subsequently, reactions with MPs with and without hydrazide groups were investigated. Finally, various carboxyl group-containing MPs were functionalized with $\mathrm{ADH}$ (Figure 1) and the amount of hydrazide groups was determined by BCA and verified by the TNBS test [18].

\section{Materials and Methods}

2.1. Chemicals. ADH, $N$-(3-dimethylaminopropyl)- $N$-ethylcarbodiimide hydrochloride (EDC), and TNBS were purchased from Sigma-Aldrich (St. Louis, MO, USA). A Pierce BCA Protein Assay Kit and $N$-hydroxysulfosuccinimide (S-NHS) were purchased from Thermo Fisher Scientific (Waltham, MA, USA). Glycine, potassium tetraborate tetrahydrate, and methanol were purchased from Penta (Prague, Czech Republic).
2.2. Magnetic Particles. SiMAG-active silica MPs ( $1 \mu \mathrm{m})$ containing carboxyl and/or hydrazide groups were purchased from Chemicell (Berlin, Germany) and were used as positive and negative controls. Noncommercial microspheres of two types were produced by the Institute of Macromolecular Chemistry, Academy of Sciences of the Czech Republic (Prague, Czech Republic): magnetic poly(glycidyl methacrylate) (PGMA) particles with carboxyl groups (PGMA$\mathrm{COOH}$ ) and/or magnetic poly(2-hydroxyethyl methacrylate) (PHEMA) with hydrazide groups (PHEMA-NH-NH ${ }_{2}$ ). Both microsphere types were $4.5 \mu \mathrm{m}$ in diameter and were prepared by multistep swelling polymerization of glycidyl methacrylate (GMA) and/or 2-hydroxyethyl methacrylate (HEMA) with a carboxyl-containing monomer [19] followed by precipitation of iron oxides inside the pores [20, 21]. Hydrazide-containing nonmagnetic bead cellulose (80$100 \mu \mathrm{m}$ in diameter) was used as a standard with declared hydrazide group content $(15 \mu \mathrm{mol} / \mathrm{mL})$ [22].

2.3. Modified TNBS Test. The protocol for hydrazide quantification was based on a previously published method [18], but the reaction volumes were decreased 10-fold. An aliquot of MPs (1-5 mg) was washed three times with distilled water using a DynaMag magnetic separator (Life Technologies, Carlsbad, CA, USA), and $900 \mu \mathrm{L}$ of $0.1 \mathrm{M}$ potassium tetraborate tetrahydrate and $100 \mu \mathrm{L}$ of $3 \mathrm{mM}$ TNBS were added. The same protocol without the particles was used for the reference blank. Mixtures were incubated at $37^{\circ} \mathrm{C}$ for $2 \mathrm{~h}$ with rotation. After incubation, $500 \mu \mathrm{L}$ of $0.1 \mathrm{M}$ potassium tetraborate tetrahydrate and $100 \mu \mathrm{L}$ of particle supernatant were added to two new tubes. Then, $50 \mu \mathrm{L}$ of $30 \mathrm{mM}$ glycine was added to the first tube (sample), while $50 \mu \mathrm{L}$ of distilled water was added to the second tube (sample blank). Incubation at room temperature (RT) for $25 \mathrm{~min}$ with rotation followed, and $1 \mathrm{~mL}$ of cold methanol was added to each tube. Absorbance was measured in a $1 \mathrm{~cm}$ quartz cuvette at $340 \mathrm{~nm}$ against the blank on a Libra S22 UV/VIS spectrophotometer (Biochrom, Cambridge, UK).

2.4. BCA Assay. An aliquot of MPs (1-5 mg) was washed $1 \mathrm{x}$ with $1.5 \mathrm{~mL}$ distilled water. The BCA reagent (BCA containing the $\mathrm{Cu}^{2+}$ ions) was prepared according to the recommendations of the BCA kit's supplier. Then, $0.5 \mathrm{mg}$ of MPs was resuspended in $500 \mu \mathrm{L}$ of the reagent, and the mixture was incubated at $60^{\circ} \mathrm{C}$ for $1 \mathrm{~h}$ with rotation. After incubation, MPs were separated using a magnetic separator and the 


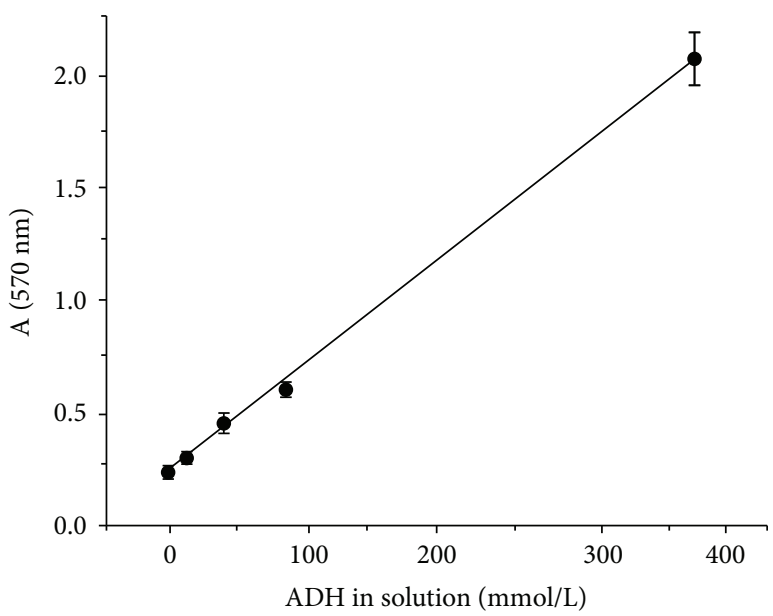

(a)

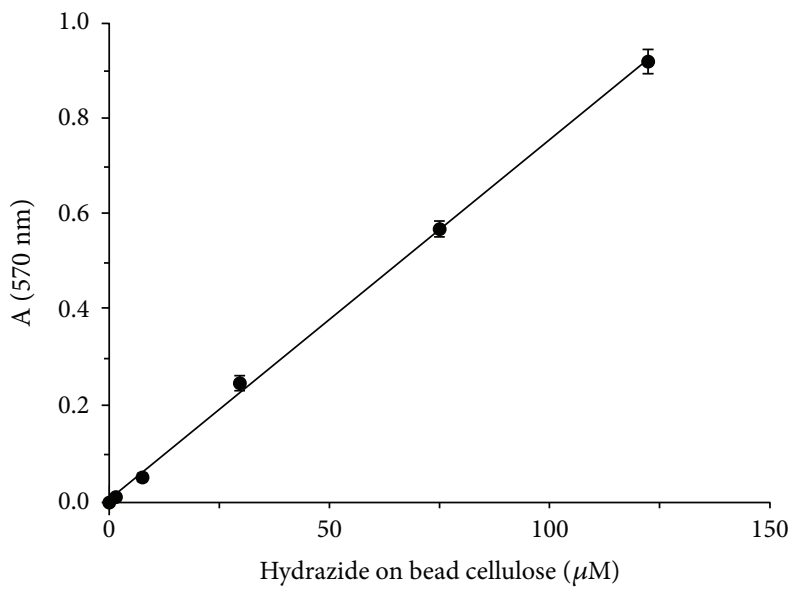

(c)

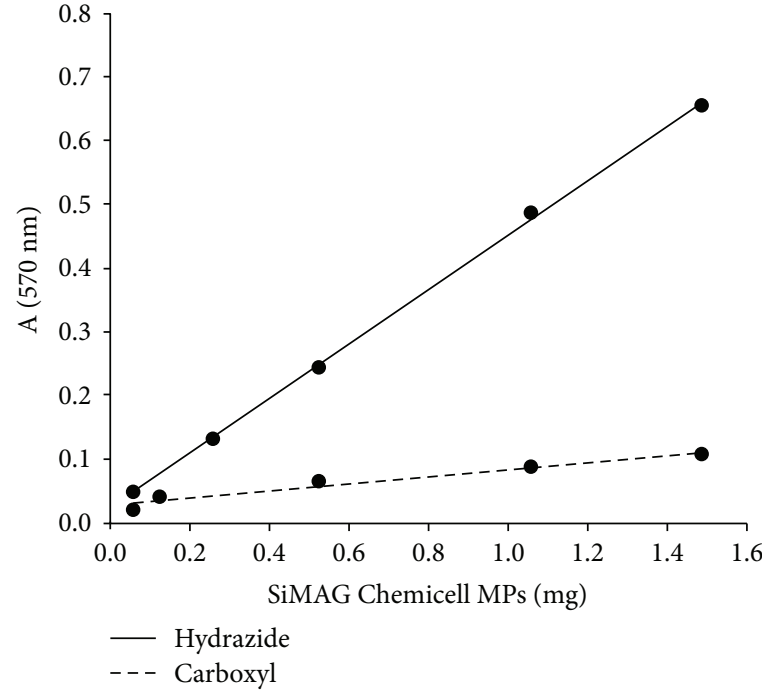

(b)

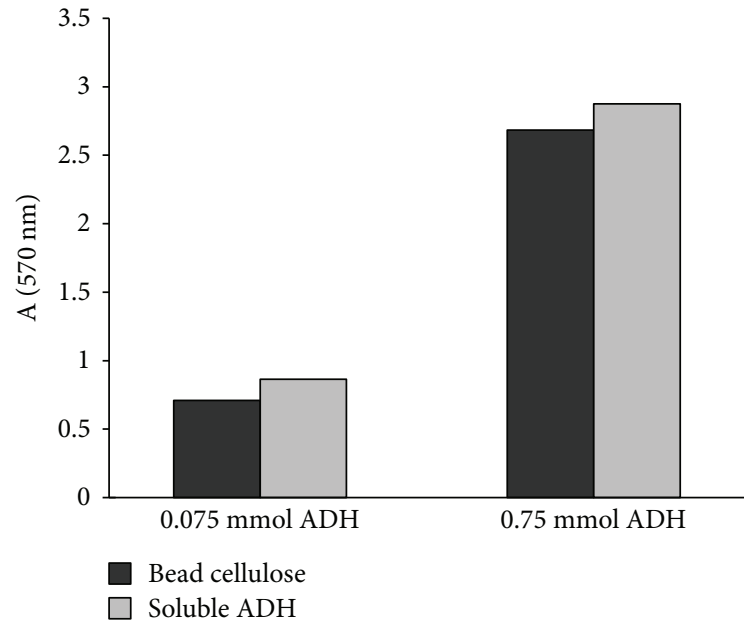

(d)

FIGURE 2: The colorimetric determination of hydrazide groups using the BCA reagent. (a) Linear correlation of absorbance (570 nm) and $\mathrm{ADH}$ solution in the concentration range of $0.022-2.2 \mathrm{mmol} / \mathrm{L}\left(R^{2}=0.999\right)$. (b) Standard curves obtained by the reaction of BCA with various amounts of hydrazide- and/or carboxyl-containing MPs $\left(0-1.5 \mathrm{mg}, R^{2}=0.998\right)$. (c) Standard curve obtained after the reaction of various amounts of hydrazide bead cellulose $(1.2-120 \mu \mathrm{mol} / \mathrm{L})$ with BCA $\left(R^{2}=0.999\right)$. (d) The comparison of absorbance from hydrazide bead cellulose $(10$ and $100 \mu \mathrm{L})$ and their equimolar amounts of standard $\mathrm{ADH}$ solutions $(0.075$ and $0.75 \mathrm{mmol})$.

TABle 1: Magnetic particles used for the determination of hydrazide groups.

\begin{tabular}{lcc}
\hline Magnetic particles & Functional group & Characteristics \\
\hline \multirow{2}{*}{ SiMAG $(1 \mu \mathrm{m})$} & Hydrazide & Positive control \\
& Carboxyl & Negative control \\
& Carboxyl + ADH & ADH-functionalized \\
\hline \multirow{2}{*}{ PHEMA $(4.5 \mu \mathrm{m})$} & Hydrazide & Positive control* \\
& Carboxyl & Negative control \\
& Carboxyl + ADH & ADH-functionalized \\
\hline \multirow{2}{*}{ PGMA $(4.5 \mu \mathrm{m})$} & Carboxyl & Negative control \\
& Carboxyl + ADH & ADH-functionalized \\
\hline
\end{tabular}

*Amount of hydrazide groups declared by supplier. ADH: adipic acid dihydrazide; PGMA: poly(glycidyl methacrylate); PHEMA: poly(2hydroxyethyl methacrylate). absorbance of $200 \mu \mathrm{L}$ supernatant was measured in a microtitration plate at $570 \mathrm{~nm}$ against the reagent blank using a Multiskan RC reader from Labsystems (Franklin, MA, USA).

2.5. Functionalization of Carboxyl-Containing Particles by $A D H$. A magnetic carrier containing carboxyl groups $(1 \mathrm{mg})$ was washed three times with $1.5 \mathrm{~mL}$ of $10 \mathrm{mM}$ phosphate buffer ( $\mathrm{pH} 6.0)$ and activated by zero-crosslinker EDC and S-NHS. MPs were mixed with $3 \mathrm{mg}$ S-NHS and $4 \mathrm{mg}$ EDC dissolved in $500 \mu \mathrm{L}$ of $10 \mathrm{mM}$ phosphate buffer ( $\mathrm{pH}$ 6.0). The suspension was incubated at RT for $15 \mathrm{~min}$ with rotation, and the MPs were washed with $1 \mathrm{~mL}$ of $2 \mathrm{mM} \mathrm{HCl}$. The MP pellet was immediately resuspended in $500 \mu \mathrm{L}$ of $20 \mathrm{mM}$ phosphate buffer (pH 7.5) with $6 \mathrm{mg} \mathrm{ADH}$ and incubated at RT for $2 \mathrm{~h}$ with rotation. Functionalized 


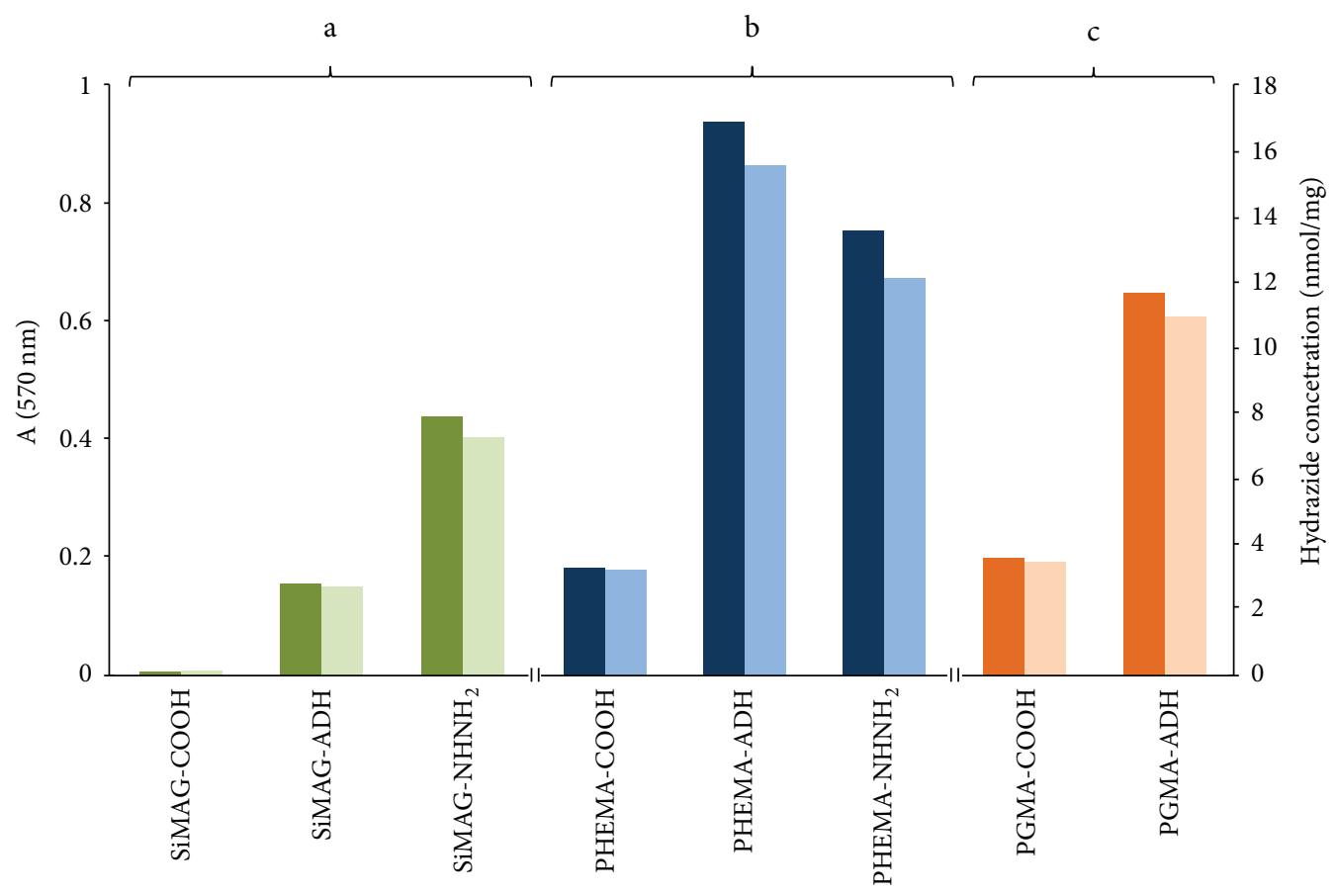

FIGURE 3: The comparison of two methods for the determination of hydrazide groups. TNBS (dark columns) and BCA-based (light columns) tests on three carboxyl-containing MPs functionalized with ADH. (a) SiMAG, (b) PHEMA, and (c) PGMA. Particles with carboxyl groups served as negative controls, and those with hydrazide groups as positive controls.

MPs were washed three times with $1.5 \mathrm{~mL}$ of $20 \mathrm{mM}$ phosphate buffer ( $\mathrm{pH} 7.5)$.

\section{Results and Discussion}

3.1. Quantification of Hydrazide Groups on Magnetic Particles Using BCA Reagent. The first experiments were designed to confirm the assumption that the BCA reagent may be used to estimate the amount of hydrazide groups on MPs. A standard ADH solution in a concentration range of two orders of magnitude $(0.022-2.2 \mathrm{mmol} / \mathrm{L})$ was investigated. The absorbance level at $570 \mathrm{~nm}$ linearly correlated with the molarity of ADH (Figure 2(a)). The use of the standard ADH solution should eliminate the potential influence of the magnetic solid support and generate a signal coming only from the hydrazide groups.

In the next step, Chemicell MPs with or without hydrazide groups (optionally with carboxyl groups) served as a standard in investigating the significance of differences in their absorbance signals. A strong linear correlation was observed between absorbance mediated by the BCA reagent and an increasing amount of hydrazide MPs $(0-1.5 \mathrm{mg}$; Figure 2(b), dashed line). On the other hand, and as expected, the signal from carboxyl MPs (continuous line in Figure 2(b)) almost did not change with an increasing amount of the hydrazide carrier (absorbance at $570 \mathrm{~nm}$ was 0.07 per mg of MPs).

To quantify the amount of hydrazide groups on the particles, the interaction between the nonmagnetic macroporous bead cellulose and a declared amount of hydrazide groups $(15 \mu \mathrm{mol} / \mathrm{mL})$ was also studied. Various amounts of packed beads $(0.08,0.4,2,4$, and $8 \mu \mathrm{L})$ contained $1.2,6$, 30,60 , and $120 \mu \mathrm{mol} / \mathrm{L}$ of hydrazide groups (Figure 2(c)). Absorbance increased linearly with an increasing amount of the hydrazide carrier.

Subsequently, the absorbance from the hydrazide groups on bead cellulose ( 10 and $100 \mu \mathrm{L}$ ) with functional group concentrations of 0.075 and $0.75 \mathrm{mmol}$ was compared with the signal from the $\mathrm{ADH}$ solution with the same concentration of hydrazide groups as on the bead cellulose (Figure 2(d)). The absorbances from both samples were in accord for both concentrations.

\subsection{Hydrazide Functionalization of Carboxyl-Containing} Magnetic Particles. Three various MPs with carboxyl groups were used for hydrazide functionalization with $\mathrm{ADH}$ : SiMAG-carboxyl MPs, PGMA, and PHEMA MPs. The carboxyl-containing MPs were used as a negative control, and the MPs with a defined amount of hydrazide groups were used as a positive control. The newly tested technique with BCA and the conventional TNBS test were subsequently compared for the determination of hydrazide groups on the MPs mentioned in Table 1.

3.3. Determination of Hydrazide Groups on ADHFunctionalized MPs. In this experiment, various carboxylcontaining MPs were functionalized with hydrazide groups. The amounts of the hydrazide groups on the MPs and their controls (Table 1) were analysed using the newly tested BCA-based technique as well as the TNBS test routinely used for the determination of hydrazide groups. The method is based on the functional groups' reaction with excess TNBS 
and subsequent quantification of residual unreacted TNBS by reaction with glycine. The original protocol [18] was modified and optimized for the MPs. Moreover, the reaction volumes of toxic reagents, such as TNBS and methanol, were significantly reduced.

The results from both tests performed on MPs from Table 1 (presented in Figure 3) were closely correlated, as indicated by the paired Student $t$-test $(P=0.0018)$ and the coefficient of determination (0.98). The importance of the negative controls, which were close to zero for SiMAG but 3.23 and $3.46 \mu \mathrm{mol} / \mathrm{g}$ for the PHEMA and PGMA MPs, respectively, was confirmed. Each matrix interacts with the BCA reagent individually, and so MPs must be explored on a case-by-case basis. It has been repeatedly established that determining the difference between the signal of the functionalized MPs and the signal of the appropriate negative control enables precise quantification as to the amount of bound hydrazide groups. The quantity of hydrazide groups was $2.74 \mu \mathrm{mol} / \mathrm{g}$ of SiMAG, $16.50 \mu \mathrm{mol} / \mathrm{g}$ of PHEMA MPs, and $11.41 \mu \mathrm{mol} / \mathrm{g}$ of PGMA-EDMA MPs. Such particles with estimated amounts of hydrazide functional groups could subsequently be applied to site-specific immobilization of glycoproteins.

\section{Conclusions}

A simple, one-step, rapid, low-toxic method for the quantitative determination of hydrazide groups on solid support using the BCA reagent was evaluated on various MPs, including macroporous bead cellulose, polymer MPs, and nonporous silica MPs. The conventional method using BCA for protein quantification in liquid media was here applied for the determination of hydrazide groups presented on magnetic spherical particles. This approach was successfully used for the evaluation of hydrazide functionalization of carboxyl group-containing MPs (PHEMA-COOH, PGMA-COOH, and SiMAG). The obtained results were confirmed using the previously established TNBS test. This work verified that the analytical technique based on BCA is suitable for the determination of hydrazide functional groups on MPs, which subsequently can be utilized for site-specific conjugation of glycoproteins and/or other bioapplications.

\section{Conflicts of Interest}

The authors declare that they have no conflicts of interest.

\section{Acknowledgments}

The authors would like to acknowledge the financial support of the European Commission through HORIZON2020-ICT 28-2015 grant (LoveFood2Market, no. 687681).

\section{References}

[1] A. R. Varlan, J. Suls, P. Jacobs, and W. Sansen, "A new technique of enzyme entrapment for planar biosensors," Biosensors and Bioelectronics, vol. 10, no. 8, pp. xv-xix, 1995.
[2] M. K. Jones and J. Balen, "Magnetic beads for schistosomiasis diagnosis," PLoS Neglected Tropical Diseases, vol. 1, no. 3, p. e159, 2007.

[3] M. Liu, C. Jia, Y. Huang et al., The Analyst, vol. 135, no. 2, pp. 327-331, 2010.

[4] Z.-M. Liu, H.-F. Yang, Y.-F. Li, Y.-L. Liu, G.-L. Shen, and R.Q. Yu, Sensors and Actuators B: Chemical, vol. 113, no. 2, pp. 956-962, 2006.

[5] C. C. Berry and A. S. G. Curtis, "Functionalisation of magnetic nanoparticles for applications in biomedicine," Journal of Physics D: Applied Physics, vol. 36, no. 13, pp. R198-R206, 2003.

[6] J.-M. Kauffmann, D. Yu, A. El Yacoubi, and B. Blankert, "Magnetic nanoporous microparticles for biosensors and bioreactors," LabPlus International, vol. 20, no. 3, p. 6, 2006.

[7] J. Dobson, Nanomedicine, vol. 1, no. 1, pp. 31-37, 2006.

[8] B. Solomon, R. Koppel, F. Schwartz, and G. Fleminger, Journal of Chromatography. A, vol. 510, pp. 321-329, 1990.

[9] R. L. Wimalasena and G. S. Wilson, Journal of Chromatography B: Biomedical Sciences and Applications, vol. 572, no. 12, pp. 85-102, 1991.

[10] S. V. Rao, K. W. Anderson, and L. G. Bachas, Microchimica Acta, vol. 128, no. 3-4, pp. 127-143, 1998.

[11] J. H. Kang, H. J. Choi, S. Y. Hwang, S. H. Han, J. Y. Jeon, and E. K. Lee, Journal of Chromatography. A, vol. 1161, no. 1-2, pp. 9-14, 2007.

[12] P. K. Smith, R. I. Krohn, G. T. Hermanson et al., Analytical Biochemistry, vol. 150, no. 1, pp. 76-85, 1985.

[13] K. J. Wiechelman, R. D. Braun, and J. D. Fitzpatrick, Analytical Biochemistry, vol. 175, no. 1, pp. 231-237, 1988.

[14] R. J. Kessler and D. D. Fanestil, Analytical Biochemistry, vol. 159, no. 1, pp. 138-142, 1986.

[15] S. Kakabakos, P. Tyllianakis, G. Evangelatos, and D. Ithakissios, Biomaterials, vol. 15, no. 4, pp. 289-297, 1994.

[16] P. E. Tyllianakis, S. E. Kakabakos, G. P. Evangelatos, and D. S. Ithakissios, Analytical Biochemistry, vol. 219, no. 2, pp. 335340, 1994.

[17] Z. Qu, H. Xu, P. Ning, and H. Gu, Surface and Interface Analysis, vol. 44, no. 10, pp. 1309-1313, 2012.

[18] G. Antoni, R. Presentini, and P. Neri, Analytical Biochemistry, vol. 129, no. 1, pp. 60-63, 1983.

[19] J. Ugelstad, T. Ellingsen, A. Berge, and O. B. Helgee, "Magnetic polymer particles and process for the preparation thereof," 1987, US Patent 4654267.

[20] D. Horák, B. Rittich, J. Šafář, A. Španová, J. Lenfeld, and M. J. Beneš, Biotechnology Progress, vol. 17, no. 3, pp. 447-452, 2001.

[21] D. Horák and N. Chekina, Journal of Applied Polymer Science, vol. 102, no. 5, pp. 4348-4357, 2006.

[22] M. J. Beneš, K. Adámková, and J. Turková, “Activation of beaded cellulose with 2, 4, 6-trichlorotriazine," Journal of Bioactive and Compatible Polymers, vol. 6, p. 406, 1991. 

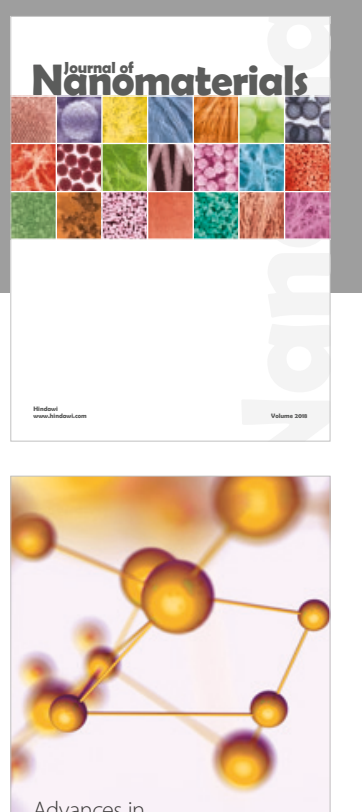

Physical Chemistry
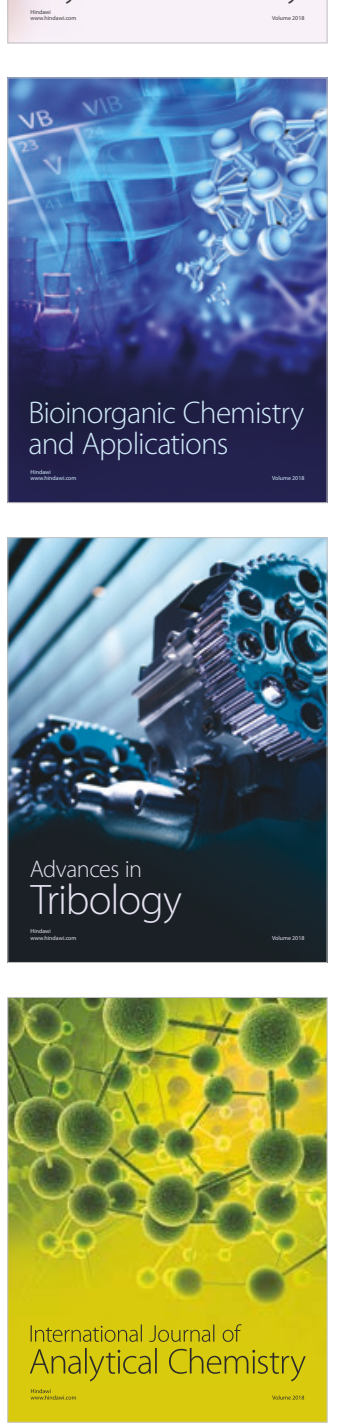

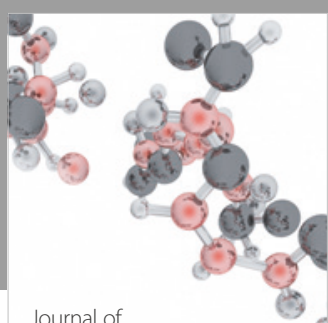

Analytical Methods

in Chemistry

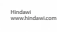

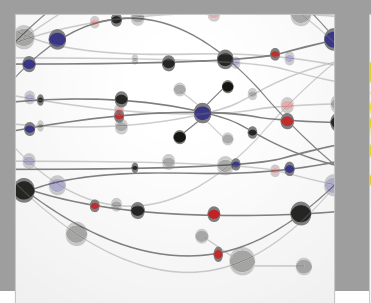

The Scientific World Journal

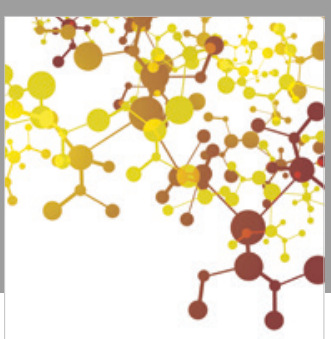

Journal of

Applied Chemistry
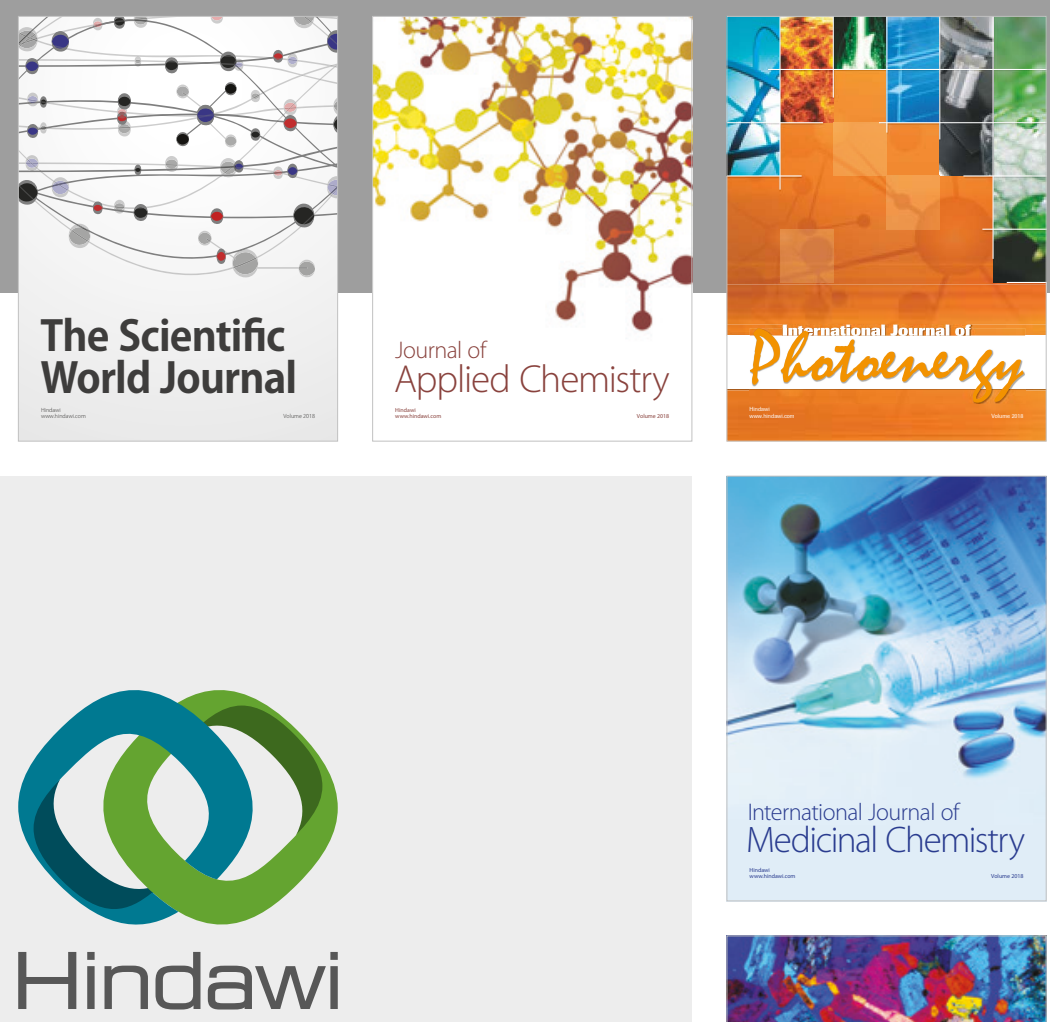

Submit your manuscripts at

www.hindawi.com
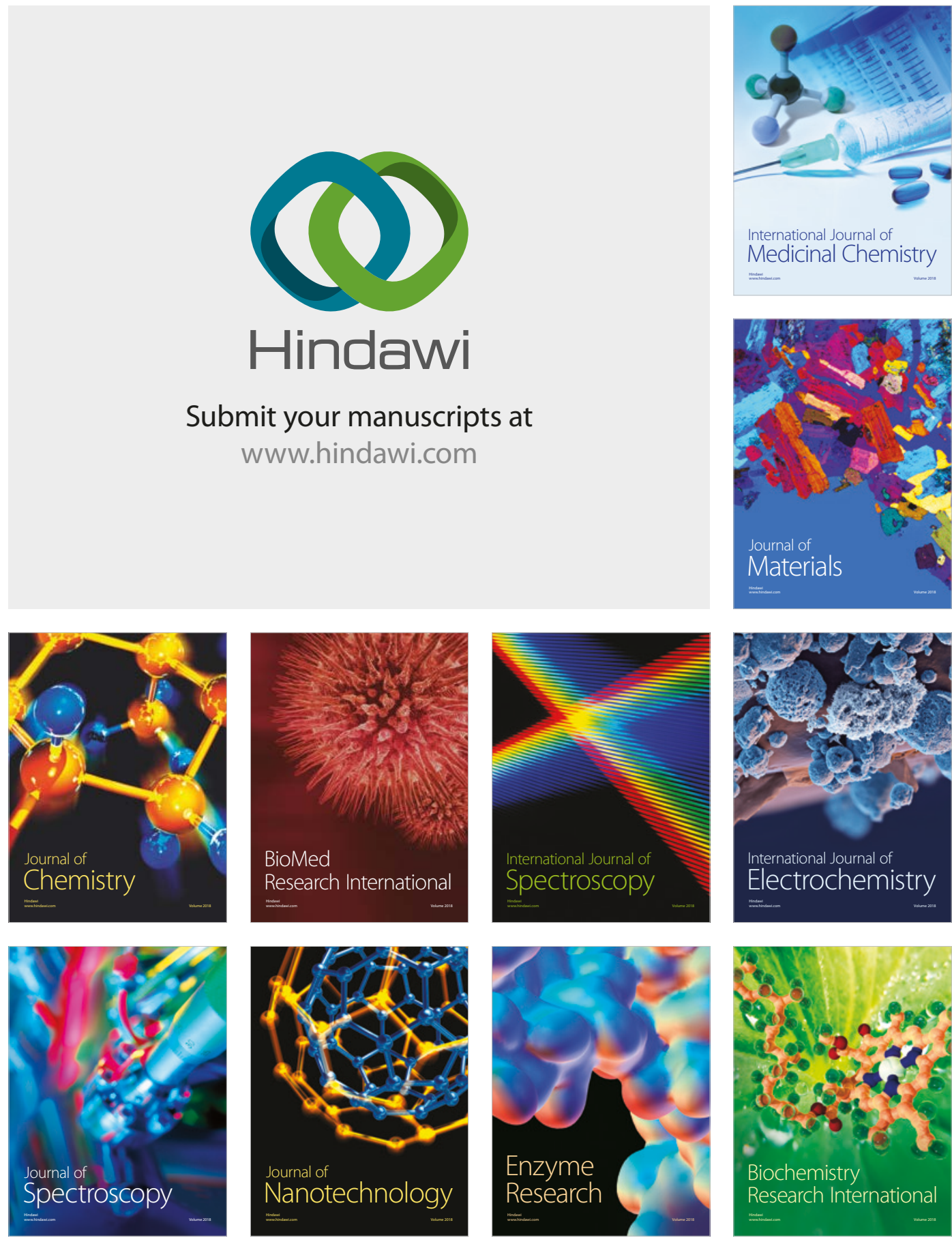
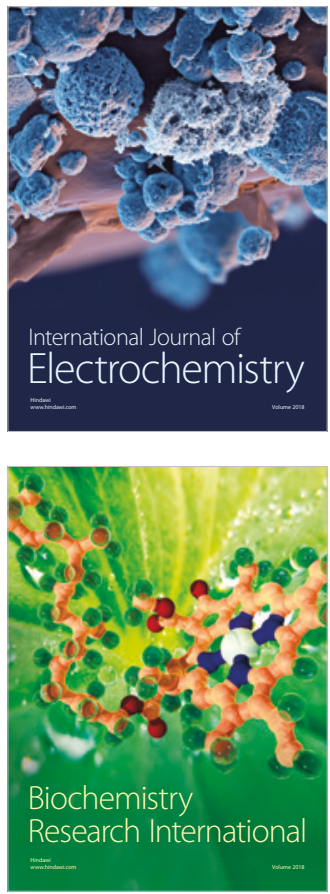